\title{
Stapes ankylosis with broad thumbs and toes
}

INSERM

\section{Source}

INSERM. (1999). Orphanet: an online rare disease and orphan drug data base. Stapes ankylosis with broad thumbs and toes. ORPHA:140917

Stapes ankylosis with broad thumbs and toes is a very rare genetic bone disorder characterized by ankylosis of stapes, broad thumbs and halluces, conductive hearing loss and hyperopia. 